\title{
CORROSION INDUCED CRACKING OF REINFORCED CONCRETE
}

\author{
CAROLINE FAHY*, DOMENICO GALLIPOLI ${ }^{\dagger}$ AND PETER GRASSL ${ }^{*}$ \\ *University of Glasgow \\ Oakfield Avenue, Glasgow, UK \\ e-mail: c.fahy.1@research.gla.ac.uk, peter.grassl@glasgow.ac.uk \\ †Université de Pau et des Pays de l’Adour, \\ Laboratoire SIAME, France \\ email: domenico.gallipoli@univ-pau.fr
}

Key words: Corrosion Induced Cracking, Reinforced Concrete, Lattice Model, Computational Modelling

\begin{abstract}
In this work a hygro-mechanical approach to modelling corrosion induced cracking is proposed. A coupled transport and mechanical lattice model based on Delaunay and Voronoi tessellations is used to model the penetration of corrosion products into a concrete specimen. The penetration of the corrosion products into the concrete is described by constitutive laws developed for mass transport through porous materials. The cracking caused by the penetration of corrosion products is modelled by the mechanical lattice using a damage mechanics constitutive law. The coupled approach is verified by comparison of the numerical results with the analytical solution of an elastic thick-walled permeable cylinder, which has been derived in the present work. Then, the coupled model is applied to the analysis of corrosion induced cracking. The results of the new approach are compared to those of a purely mechanical approach for an impermeable material.
\end{abstract}

\section{INTRODUCTION}

Corrosion induced cracking and spalling has a major influence on the life of reinforced concrete structures and is caused by the constrained volume expansion of corroded reinforcement bars. In previous work, the process of corrosion induced cracking has been commonly modelled by a mechanical approach using an expansive layer between the steel and the concrete [1]. In the present work an alternative approach to modelling corrosion induced cracking is proposed. The penetration of corrosion products into concrete is modelled by a mass transport lattice model for fully saturated materials which is coupled to a mechanical lattice approach. By assuming that the product generated by corrosion is a fluid permeating the porous concrete and by following the theory of effective stresses
[2], the fluid pressure obtained from the transport lattice is added to the normal stress in the mechanical model. Lattice models are suitable for this type of work as they have been shown to describe well displacement discontinuities that occur in fracture processes [3] and to accurately model flow in porous materials [4]. Cracking is modelled by a damage mechanics constitutive law and transport is described by Darcy's law.

\section{LATTICE MODEL}

In the present work, fluid transport and mechanical response are modeled by lattices based on one-dimensional structural and transport elements. The domain is discretised by a dual Delaunay and Voronoi tessellation. Figure 1 shows the structure of this dual lattice mesh. For the mechanical model, the lattice elements 
are placed along the edges of the Delaunay triangles [4]. The geometry of the mid crosssection of the elements is determined by the corresponding Voronoi polygon edges, (Figure 2). The transport lattice consists of onedimensional transport elements placed along the edges of the Voronoi polygons [3]. Their corresponding cross-sectional properties are determined from the corresponding edges of the Delaunay triangles. The point $C$ in Figure 2 contains information for both the mechanical and the transport model, which simplifies the coupling of the two models.

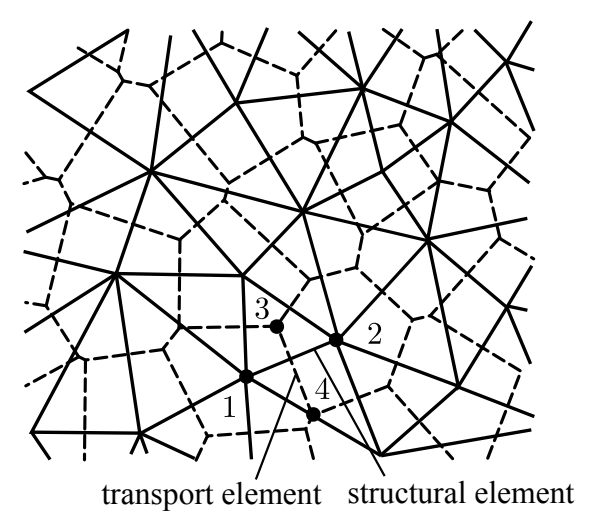

Figure 1: Dual lattice mesh.
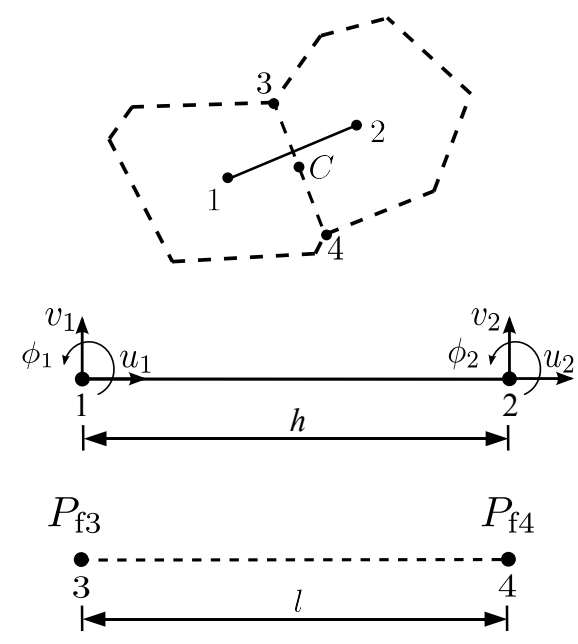

Figure 2: Dual lattice elements.

In Figure 2, $u, v$ and $\phi$ are nodal degrees of freedom of the mechanical model. Furthermore, $P_{\mathrm{f}}$ is the nodal unknown (fluid pressure) of the transport model.

\subsection{Mechanical Model}

The stress of the mechanical model which enters the equilibrium condition is split into an effective stress carried by the solid and the fluid pressure in the pores by applying the principle of effective stress used in soil mechanics:

$$
\boldsymbol{\sigma}=\boldsymbol{\sigma}^{\mathrm{m}}+\boldsymbol{\sigma}^{\mathrm{f}}
$$

where $\boldsymbol{\sigma}=\left\{\sigma_{\mathrm{n}}, \sigma_{\mathrm{s}}, \sigma_{\phi}\right\}^{T}$ is the stress vector (comprising of normal, shear and rotational stresses), $\boldsymbol{\sigma}^{\mathrm{m}}=\left\{\sigma_{\mathrm{n}}^{\mathrm{m}}, \sigma_{\mathrm{s}}^{\mathrm{m}}, \sigma_{\phi}^{\mathrm{m}}\right\}^{T}$ is the effective stress vector and $\boldsymbol{\sigma}^{\mathrm{f}}=\left\{P_{\mathrm{f}}, 0,0\right\}^{T}$ is the fluid pressure vector (see also Section 2.2).

For the effective stress, an isotropic damage model is used to describe the inelastic response of the lattice element which corresponds to a continuous reduction of the element's stiffness. The stress-strain law for this part is

$$
\boldsymbol{\sigma}^{\mathrm{m}}=(1-\omega) \mathbf{D}: \boldsymbol{\varepsilon}
$$

where $\omega$ is a damage parameter, $\mathbf{D}$ is the elastic stiffness and $\varepsilon=\left\{\varepsilon_{\mathrm{n}}, \varepsilon_{\mathrm{s}}, \varepsilon_{\phi}\right\}^{T}$ is the strain vector (comprising of normal, shear and rotational strains). The elastic stiffness is defined as

$$
\mathbf{D}=\left[\begin{array}{ccc}
E & 0 & 0 \\
0 & \gamma E & 0 \\
0 & 0 & E
\end{array}\right]
$$

and depends on the model parameters $E$ and $\gamma$. For plane stress analysis and a regular lattice of equilateral triangles, these model parameters are related to the continuum Young's modulus $E_{\mathrm{c}}$ and Poisson's ratio $\nu$ as

$$
\begin{gathered}
\gamma=\frac{1-3 \nu}{\nu+1} \\
E=\frac{E_{\mathrm{c}}}{1-\nu}
\end{gathered}
$$

For the irregular lattices used in this study the expressions in (4) and (5) are used as an approximation.

The damage parameter $\omega$ in (2) is a function of a history variable $\kappa$ which is determined from the loading function

$$
f(\varepsilon, \kappa)=\varepsilon_{\text {eq }}(\varepsilon)-\kappa
$$

and the loading-unloading conditions

$$
f \leq 0, \quad \dot{\kappa} \geq 0, \quad \dot{\kappa} f=0
$$


The equivalent strain

$$
\begin{aligned}
\varepsilon_{\mathrm{eq}} & =\frac{1}{2} \varepsilon_{0}(1-c)+ \\
& +\sqrt{\left(\frac{1}{2} \varepsilon_{0}(c-1)+\varepsilon_{\mathrm{n}}\right)^{2}+\frac{c \gamma^{2} \varepsilon_{\mathrm{s}}^{2}}{q^{2}}}
\end{aligned}
$$

corresponds to an elliptical strength envelope for $\varepsilon_{\text {eq }}=\varepsilon_{0}=f_{\mathrm{t}} / E$ (Figure 3). Here, $f_{\mathrm{t}}$ is the tensile strength of the lattice material, $c$ is a parameter which relates the compressive strength to the tensile strength and $q$ relates the shear strength to the tensile strength.

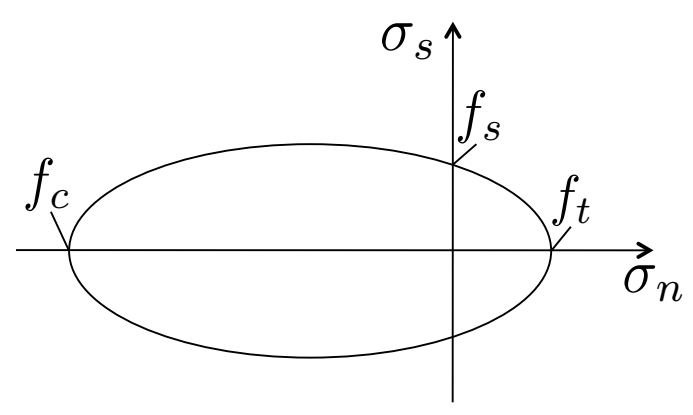

Figure 3: Elliptical strength envelope.

The damage function is chosen so that for normal tensile loading $\left(\sigma_{\mathrm{s}}^{\mathrm{m}}=\sigma_{\phi}^{\mathrm{m}}=0\right)$ an exponential stress crack opening law of the form

$$
\sigma_{\mathrm{n}}^{\mathrm{m}}=f_{\mathrm{t}} \exp \left(-\frac{w_{\mathrm{cn}}}{w_{\mathrm{f}}}\right)
$$

is obtained, where $w_{\mathrm{cn}}$ is the normal crack opening and $w_{\mathrm{f}}$ is the crack opening threshold at which the material is fully damaged $(\omega=1)$.

\subsection{Transport Model}

The mass balance equation presented in this section describes the stationary mass flow through a transport element subjected to fluid pressure gradient. The material is assumed to be fully saturated. In the balance equation, the flux (i.e. the rate of liquid flow per unit area) is related to the pressure gradient through Darcy's law, which gives

$$
k \operatorname{div}\left(\operatorname{grad}\left(P_{\mathrm{f}}\right)\right)=0
$$

where $k$ is the hydraulic conductivity, $P_{\mathrm{f}}$ is the fluid pressure. The hydraulic conductivity is defined as $k=\rho \kappa / \mu$ where $\rho$ is the density of the fluid, $\kappa$ is the intrinsic permeability of the porous material and $\mu$ is the absolute (dynamic) viscosity of the fluid.

Boundary conditions are imposed either as prescribed values of fluid pressure (on boundary $\Gamma_{1}$ ) or as prescribed values of flux (on boundary $\Gamma_{2}$ ). The latter boundary condition can then be related to the gradient of fluid pressure through Darcy's law. This results in the following two mathematical constraints on $P_{\mathrm{f}}$ :

$P_{\mathrm{f}}=g(\mathbf{x})$ on $\Gamma_{1} \quad$ and $\quad-\frac{\partial P_{\mathrm{f}}}{\partial \mathbf{n}}=f(\mathbf{x})$ on $\Gamma_{2}$

where $\mathbf{n}$ denotes the direction normal to the boundary while $g(\mathbf{x})$ and $f(\mathbf{x})$ are functions of the spatial coordinate vector $\mathrm{x}$. More details on the discretised form of these equations can be found in [6].

\section{ANALYSIS OF THICK-WALLED CYLINDER}

The potential of the coupled lattice approach to describe the interaction of transport and mechanical responses was investigated by analysing an elastic plane stress thick-walled permeable cylinder subjected to an internal and external pressure (Figure 4). The response of the permeable cylinder is also compared to the behaviour of the impermeable one.

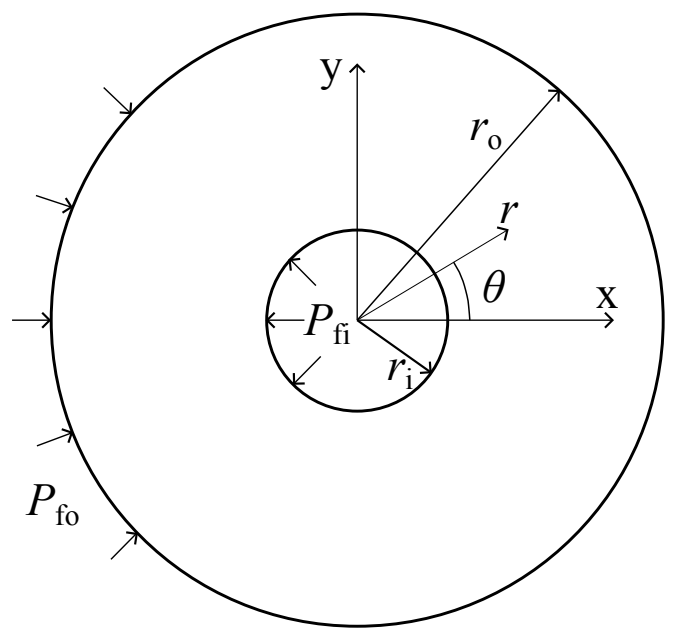

Figure 4: Geometry of the thick-walled cylinder.

The numerical solutions are compared to an analytical equation for the radial displacements of an elastic thick-walled permeable cylinder, 
which were derived in the present study. The present case is limited to a stationary flow problem with homogeneous properties for both the fluid and the solid. Other, more general, work on thick-walled permeable cylinders has been published in [8,9].

Firstly, the radial fluid pressure distribution is determined. The flux (i.e. flow rate per unit area) $q$ in the radial direction is

$$
q=\frac{Q}{2 \pi r t}
$$

and in terms of conductivity and gradient of fluid pressure

$$
q=-\frac{k}{\mu} \frac{d P_{\mathrm{c}}}{d r}
$$

where $Q$ is the total flow rate, $r$ is the radius and $t$ is the out of plane thickness. Setting (11) and (12) equal, integrating and using the boundary conditions to determine $Q$ and the integration constant gives

$$
P_{\mathrm{f}}(r)=\frac{\ln \frac{r_{\mathrm{o}}}{r}}{\ln \frac{r_{\mathrm{o}}}{r_{\mathrm{i}}}} P_{\mathrm{fi}}+P_{\mathrm{fo}}
$$

where $P_{\mathrm{fi}}$ and $P_{\mathrm{fo}}$ are the internal and external fluid pressures, respectively (Figure 4). Secondly, the equilibrium equation is given as

$$
\frac{d \sigma_{\mathrm{r}}}{d r}+\frac{\sigma_{\mathrm{r}}-\sigma_{\theta}}{r}=0
$$

where $\sigma_{\mathrm{r}}=\sigma_{\mathrm{r}}^{\mathrm{m}}+P_{\mathrm{f}}$ is the radial stress and $\sigma_{\theta}=\sigma_{\theta}^{\mathrm{m}}+P_{\mathrm{f}}$ is the tangential stress. Using Hooke's law to relate radial and tangential stresses to the corresponding strains (see [10]), the following differential equation is obtained

$$
\frac{d^{2} u}{d r^{2}}+\frac{d u}{d r} \frac{1}{r}-\frac{u}{r^{2}}+\frac{a}{r}=0
$$

where

$$
a=-\frac{P_{\mathrm{fi}}-P_{\mathrm{fo}}}{\ln \frac{r_{\mathrm{o}}}{r_{\mathrm{i}}}} \frac{1-\nu^{2}}{E_{\mathrm{c}}}
$$

The solution of (15) for the radial displacement $u(r)$ is

$$
u(r)=-\frac{1}{2} a r \ln r+\frac{C_{1}}{r}+C_{2} r
$$

The two constants $C_{1}$ and $C_{2}$ are determined from the boundary conditions as

$$
\begin{gathered}
C_{1}=r_{\mathrm{i}}^{2} \frac{\frac{a}{2}\left[(1+\nu) \ln \frac{r_{\mathrm{i}}}{r_{\mathrm{o}}}\right]}{(\nu-1)\left(1-\left(\frac{r_{\mathrm{i}}}{r_{\mathrm{o}}}\right)^{2}\right)} \\
C_{2}=\frac{a}{2(1+\nu)}\left[1+(1+\nu) \ln r_{\mathrm{o}}\right]+ \\
+\frac{\frac{a}{2} \ln \frac{r_{\mathrm{o}}}{r_{\mathrm{i}}}}{\left(\frac{r_{\mathrm{o}}}{r_{\mathrm{i}}}\right)^{2}-1}
\end{gathered}
$$

The classical analytical solution for the radial displacements in an impermeable (without fluid pressure inside the material) thick-walled cylinder can be found in [10] and is presented here once more:

$$
\begin{aligned}
u(r) & =\frac{1-\nu}{E_{\mathrm{c}}} \frac{\left(r_{\mathrm{i}}^{2} P_{\mathrm{fi}}-r_{\mathrm{o}}^{2} P_{\mathrm{fo}}\right) r}{r_{\mathrm{o}}^{2}-r_{\mathrm{i}}^{2}}+ \\
& +\frac{1+\nu}{E_{\mathrm{c}}} \frac{\left(P_{\mathrm{fi}}-P_{\mathrm{fo}}\right) r_{\mathrm{i}}^{2} r_{\mathrm{o}}^{2}}{\left(r_{\mathrm{o}}^{2}-r_{\mathrm{i}}^{2}\right) r}
\end{aligned}
$$

The lattice model results were compared to the new analytical solution of radial displacement for the permeable cylinder and the well known solution for the impermeable cylinder [10]. For the geometry of the cylinder, the following parameters were selected: inner radius $r_{\mathrm{i}}=8 \mathrm{~mm}$, outer radius $r_{\mathrm{o}}=58 \mathrm{~mm}$, inner pressure $P_{\mathrm{fi}}=-10 \mathrm{MPa}$ and outer pressure $P_{\mathrm{fo}}=0 \mathrm{MPa}$. Furthermore, the Young's modulus of the solid was chosen as $E_{\mathrm{c}}=38.7 \mathrm{GPa}$. The response for two Poisson's ratios of $\nu=0$ and 0.2 were studied. The radial displacement is independent of the hydraulic conductivity of the fluid as long as it is constant throughout the cylinder. The comparison of the results is shown in Figure 5. The lattice model reproduces well the analytical solutions for the permeable and impermeable thick-walled cylinder. The agreement is particularly good for 


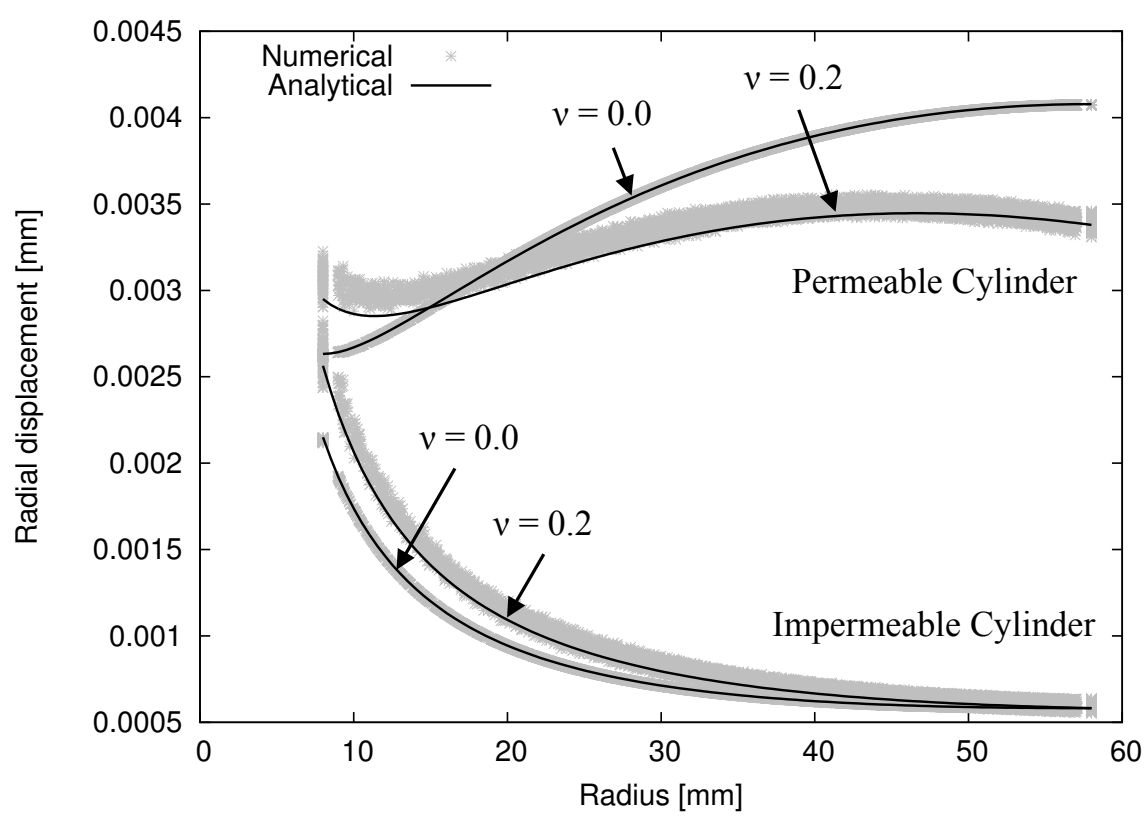

Figure 5: Comparison of numerical and analytical results for the radial displacement for the permeable and impermeable thick-walled cylinder.

$\nu=0$. The solution for the permeable thickwalled cylinder is very different from the impermeable one. For the permeable cylinder, the fluid pressure inside the material causes a stretching of the cylinder in the radial direction. On the other hand, the impermeable cylinder shortens in the radial direction. Also, Poisson's ratio has a strong influence on the result of the permeable cylinder. Not only the radial displacements, but also the stress distributions differ considerably for permeable and impermeable cylinders, which is investigated in the next section where the lattice approach is applied to the onset of corrosion induced cracking.

\section{ANALYSIS OF CORROSION IN- DUCED CRACKING}

In the present section, the new coupled lattice approach is applied to the plane stress analysis of a concrete specimen with a single eccentrically placed reinforcement bar. The geometry and material properties were taken from the experimental study on corrosion induced cracking presented in [12]. The specimen geometry is shown in Figure 6 .

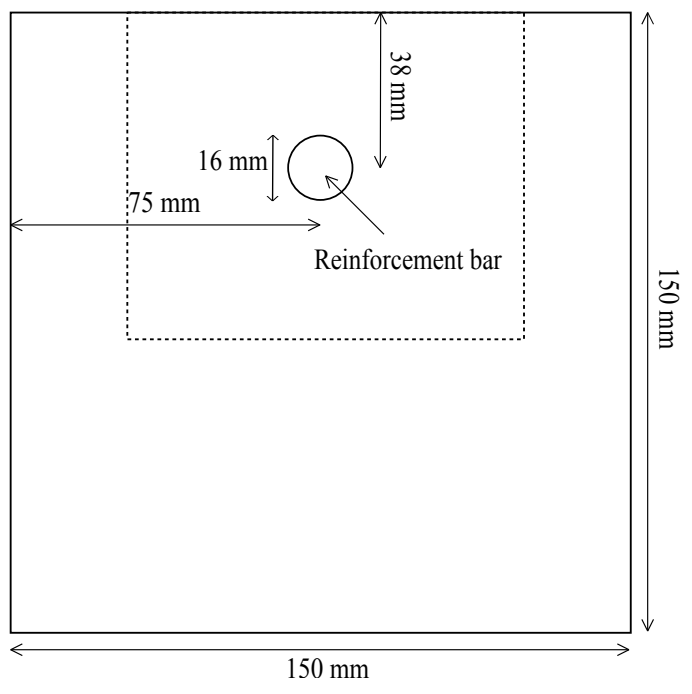

Figure 6: Geometry for concrete specimen according to [12].

In the present preliminary study the numerical results are not yet compared to the experimental ones. Instead, an internal pressure is applied at the circumference of the reinforcement bar and the response in the form of crack patterns for the permeable and impermeable specimens are compared. The material parameters for the solid were chosen as $f_{\mathrm{t}}=3.195 \mathrm{MPa}$, $E_{\mathrm{c}}=29.6 \mathrm{GPa}, \nu=0.2$. Furthermore, the fracture energy $G_{f}$ was set to a large value 


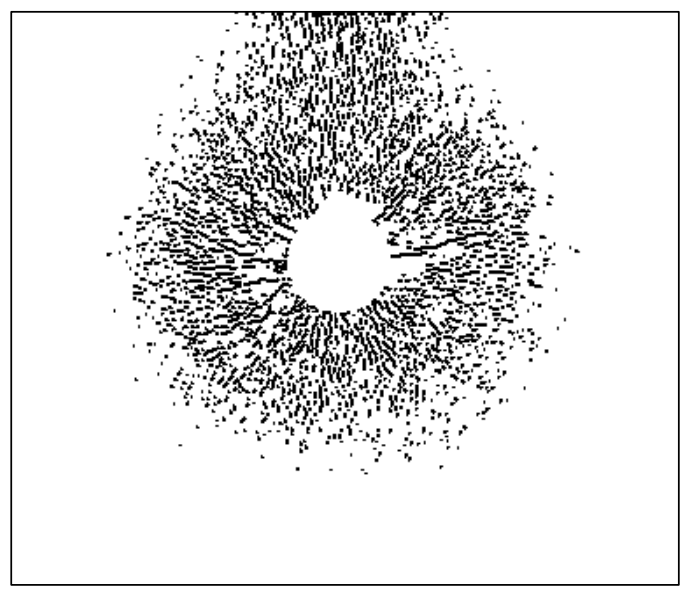

(a)

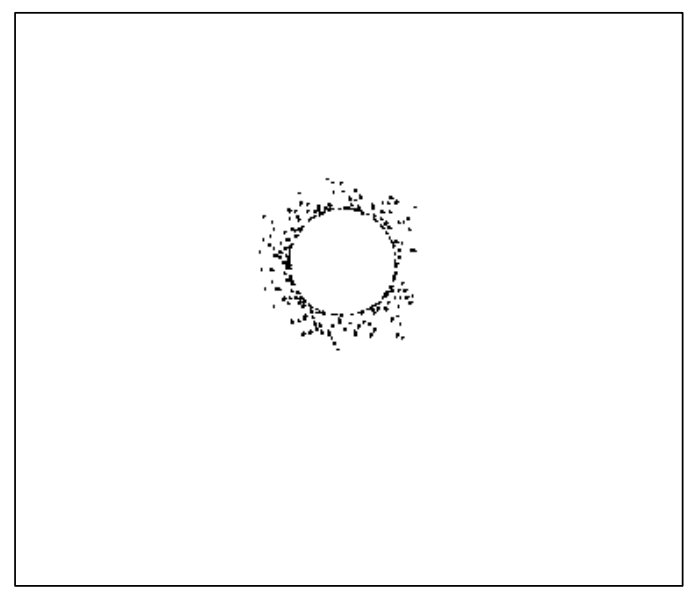

(b)

Figure 7: Detail (marked in Figure 6 by the dashed boundary) of fracture patterns for a) permeable and b) impermeable material for the same internal pressure. Black lines indicate cross-sections of mechanical elements in which damage increases at this stage of analysis.

to avoid any numerical problems in this initial study. The material parameters for the transport part were assumed as $\mu=1 \times 10^{-9} \mathrm{t} /(\mathrm{mms})$, $\kappa=1 \times 10^{-13} \mathrm{~mm}^{2}$ and $\rho=1 \times 10^{-9} \mathrm{t} / \mathrm{mm}^{3}$.

The loading in this analysis was applied as an internal pressure at the circumference of the reinforcement bar. The reinforcement bar was not modeled. The pressure distribution was determined from the transport analysis of the permeable specimen by applying a uniform flux along the circumference of the reinforcement bar. The fluid pressure at the outer boundary was assumed to be in equilibrium with the atmosphere and the specimen was assumed to be fully saturated. The flux, and therewith the pressure, was applied incrementally. Figure $7 \mathrm{a}$ shows the fracture patterns for a pressure distribution that was determined from the analysis of the permeable specimen for a flux of $1.2 \times 10^{-14} \mathrm{t} /\left(\mathrm{mm}^{2} \mathrm{~s}\right)$. For the impermeable solution the same pressure distribution was applied at the circumference of the specimen. The fracture patterns predicted for the permeable and impermeable specimen differ considerably. Significantly more cracking occurs for the permeable specimen. It should be noted that these fracture patterns are obtained for the same pressure distribution along the circumference of the reinforcement bar.

\section{CONCLUSIONS AND FUTURE WORK}

A lattice approach which couples mechanical and mass transport models using the theory of effective stresses has been applied to analyse the displacements in a thick-walled elastic cylinder and corrosion induced cracking in a concrete specimen with a single reinforcement bar. For the thick-walled elastic cylinder an analytical solution for the radial displacements has been presented. The main conclusions of the present study are the following.

For the thick-walled cylinder, the numerical and analytical results are in good agreement for both a permeable and impermeable material. For the permeable cylinder, the fluid pressure results in an increase of the thickness of the cylinder, whereas for the impermeable material the thickness decreases.

For the reinforced concrete specimen with a single reinforcement bar, the same internal pressure at the circumference of the reinforcement bar results in very different fracture patterns for permeable and impermeable materials. For the permeable material, much more cracking occurs.

In the future, the nonlinear analyses of the concrete specimen will be extended so that the analyses results can be compared to the experimental results in [12]. Also, the coupling of the mechanical and transport model will be aug- 
mented to take into account the change of conductivity due to cracking [7].

\section{ACKNOWLEDGEMENT}

The numerical analyses were performed with the nonlinear analyses program OOFEM [11] extended by the present authors.

\section{REFERENCES}

[1] Molina, F., Alonso, C. and Andrade, C., 1993. Cover cracking as a function of rebar corrosion: Part 2 - Numerical model. Materials \& Structures. 26:532-548.

[2] Terzaghi, K., 1925. Erdbaumechanik auf boden physikalischer Grundlage, Franz Deuticke.

[3] Grassl, P., 2009. A lattice approach to model flow in cracked concrete. Cement \& Concrete Composites. 31:454-460.

[4] Bolander, J.E. and Berton, S., 2004. Simulation of shrinkage induced cracking of cement composite overlays. Cement \& Concrete Composites. 26:861-871.

[5] Grassl, P. and Jirásek M., 2010. Mesoscale approach to modelling the fracture process zone of concrete subjected to uniaxial tension. International Journal of Solids and Structures. 47:957-968.

[6] Grassl, P., Grégoire, D., Rojas Solano, L. and Pijaudier-Cabot, G., 2012. Mesoscale modelling of the size effect on the fracture process zone of concrete. International Journal of Solids and Structures. 13:1818-1827.

[7] Grassl, P., Fahy C., Gallipoli, D. and Bolander J.E., 2012. A lattice model for fracture and mass transport in concrete. Proc. of The 2nd International Conference of Microdurability, April 11-13, 2012, Amsterdam, Netherlands.

[8] Shawki G. S. A. and El-Wahi S. H., 1970. Strength of thick-walled permeable cylinders. International Journal of Mechanical Sciences. 12: 535-551.

[9] Rice J. R. and Cleary M. P., 1976. Some basic stress diffusion solutions for fluidsaturated elastic porous media with compressible constituents. Reviews of Geophysics and Space Physics. 14: 227-241.

[10] Ugural, A.C. and Fenster, S.K., 1981. Advanced Strength and Applied Elasticity. The SI Version. Edward Arnold.

[11] Patzak, B. and Bittnar, Z. 2001. Design of object orientated finite element code. Advances in Engineering Software. 32:759767.

[12] Alonso, C., Andrade, C. and Molina, F., 1993. Cover cracking as a function of rebar corrosion: Part I - Experimental test. Materials \& Structures. 26:453-464.

[13] Meschke, G. and Grasberger, S., 2003. Numerical modelling of coupled hygromechanical degradation of cementitious materials. Journal of Engineering Mechanics 129:383-392.

[14] Witherspoon, P.A., Wang, J.S.Y., Jawai, K. and Gale, J.E., 1980. Validity of cubic law for fluid flow in a deformable rock structure. Water Resources Research. 16:1016-1024. 